\title{
\begin{tabular}{l|l} 
Mitraries & DSpace@MIT
\end{tabular}
}

\author{
MIT Open Access Articles
}

\section{Direct visualization of terahertz electromagnetic waves in classic experimental geometries}

The MIT Faculty has made this article openly available. Please share how this access benefits you. Your story matters.

Citation: Werley, Christopher A., Keith A. Nelson, and C. Ryan Tait. “Direct visualization of terahertz electromagnetic waves in classic experimental geometries." American Journal of Physics 80, no. 1 (2012): 72. (C) 2012 American Association of Physics Teachers.

As Published: http://dx.doi.org/10.1119/1.3652698

Publisher: American Association of Physics Teachers

Persistent URL: http://hdl.handle.net/1721.1/82595

Version: Final published version: final published article, as it appeared in a journal, conference proceedings, or other formally published context

Terms of Use: Article is made available in accordance with the publisher's policy and may be subject to US copyright law. Please refer to the publisher's site for terms of use. 


\section{AMERICAN \\ JOURNAL \\ of PHYSICS}

\section{Direct visualization of terahertz electromagnetic waves in classic experimental geometries}

Christopher A. Werley, Keith A. Nelson, and C. Ryan Tait

Citation: American Journal of Physics 80, 72 (2012); doi: 10.1119/1.3652698

View online: http://dx.doi.org/10.1119/1.3652698

View Table of Contents: http://scitation.aip.org/content/aapt/journal/ajp/80/1 ?ver=pdfcov

Published by the American Association of Physics Teachers

\section{WebAssign.}

The PREFERRED Online Homework Solution for Physics

Every textbook publisher agrees! Whichever physics text you're using, we have the proven online homework solution you need. WebAssign supports every major physics textbook from every major publisher.

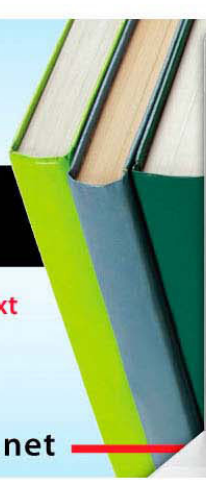

WILEY Learning:

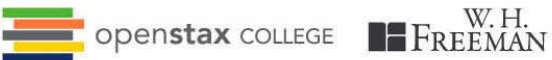

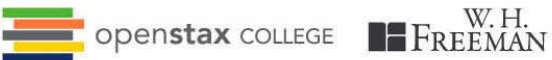
Physies Curriculum \& Instruction Higher Education PEARSON

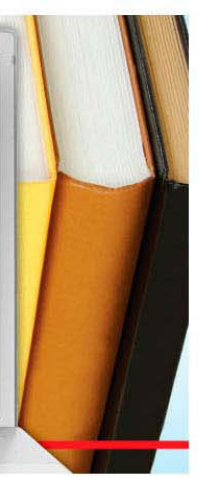




\title{
Direct visualization of terahertz electromagnetic waves in classic experimental geometries
}

\author{
Christopher A. Werley and Keith A. Nelson ${ }^{\text {a) }}$ \\ Department of Chemistry, Massachusetts Institute of Technology, Cambridge, Massachusetts 02139 \\ C. Ryan Tait \\ Department of Chemistry, Massachusetts Institute of Technology, Cambridge, Massachusetts 02139 and \\ Department of Chemistry, University of California, Berkeley, Berkeley, California 94720
}

(Received 30 March 2011; accepted 27 September 2011)

\begin{abstract}
We used newly developed experimental methods to collect educational video clips of electromagnetic waves propagating at the speed of light. The terahertz frequency waves were generated and detected in $\mathrm{LiNbO}_{3}$ crystals structured to reproduce classic optical geometries and experiments, such as two-slit interference and diffraction from a grating. Direct visualization of the phase fronts as the electromagnetic pulses propagate, reflect, diffract, and interfere helps develop intuition and understanding about the fundamental behavior of light and waves. We believe these videos will be a valuable addition to lectures on introductory optics and physics, because they bring contemporary research to the classroom and provide clear and direct experimental demonstrations of light and wave behavior. () 2012 American Association of Physics Teachers.
\end{abstract}

[DOI: 10.1119/1.3652698]

\section{INTRODUCTION}

In both optics and everyday life the behavior of light is determined by its wave-like nature, but for students of optics, this behavior can be difficult to visualize. Simple lab experiments that demonstrate wave behavior, such as diffraction or interference, usually do not enable direct visualization of the processes as they unfold or directly follow the electromagnetic wave as it interacts with objects. In Young's two-slit experiment, for example, a screen is placed far from the slits to observe an intensity pattern. The experiment is beautiful, but it does not highlight effects such as phase front curvature or interference effects close to the slits.

In this paper, we explain an experimental technique that enables the direct imaging of electromagnetic waves and the capture of short video clips of waves propagating in a nonlinear optical crystal at the speed of light. We discuss several videos of some classic optical experiments collected using this technique, which clearly show wave behavior such as reflection, focusing, interference, diffraction, and waveguiding. Our hope is that the direct experimental record of propagating electromagnetic waves will provide readers of this article and viewers of the associated videos with valuable intuition about wave behavior. We envision that the videos can most effectively be used in lectures or as supplements to lectures. For instance, after deriving the far-field diffraction pattern from two slits, an instructor could briefly explain the experimental technique (or just indicate that these are genuine, direct experimental measurements) and then show the appropriate video. It should be possible to explain the basic technique and show the selected videos in less than $5 \mathrm{~min}$, thus not using too much lecture time. Students should be encouraged to seek experimental evidence supporting the theoretical concepts they are taught, and the videos provide this evidence convincingly. We hope that, with the help of others, an online library of videos demonstrating a broad set of important optical phenomena will become available.

The video library provides students with opportunities to compare classroom discussions of wave phenomena with experimental results, which include non-ideal behavior resulting from stochastic noise fluctuations and sample imperfections. This sort of comparison is a key element of the scientific method. The ability to critically compare expectations with experimental results is important for physicists and other informed citizens when weighing issues at the intersection of science and society such as climate change and energy resources. Simulations play a complementary and equally important role. Simulations can build intuition, especially in connection with interactive programs in which users can vary parameters and observe changes in system behavior. ${ }^{1}$ In most cases simulations are inexpensive and their results are effectively free of noise, yielding clear illustrations of the behavior that results from a particular model. Most of the results in this paper can be treated analytically. Many simple geometries from introductory electricity and magnetism can also be accurately modeled with finite difference time domain (FDTD) simulations (for example, Refs. 2 and 3). The experiments here in which the wave propagates in $\mathrm{LiNbO}_{3}$ can be reproduced with a slightly more complicated formulation of the FDTD simulation which includes electromagnetic waves and nuclear motion in the crystal. ${ }^{4}$ Using conceptual formulation, analytical theory and simulation, and experimental results in concert can build competence in the specific subject being taught and in scientific thinking in general.

The frequency of the electromagnetic waves displayed in our videos is in the terahertz range, roughly $0.1-10 \mathrm{THz}$, which lies between the infrared and microwave regions of the electromagnetic spectrum. The wavelength of the $\mathrm{THz}$ waves, $\lambda=c / f \approx 30-3000 \mu \mathrm{m}$ in free space, is large enough to be easily resolved using optical imaging but small enough that many wave cycles fit within a $1 \mathrm{~cm}$ square crystal. Insight gained at terahertz frequencies can be generalized to other frequency ranges because the behavior of light has no fundamental length scale. ${ }^{5}$ Many of the phenomena we will discuss, such as diffraction, interference, reflection, and focusing, are universal for all wave types, including acoustic (sound or pressure) and water waves.

The field of terahertz research has been particularly active since about 1990 when new tools for generating and detecting terahertz radiation were developed. ${ }^{6,7}$ The first experiments on terahertz imaging took place in 1996, when terahertz 
radiation was imaged after diffracting off an object of interest. ${ }^{8,9}$ In these measurements the terahertz radiation was used for imaging of objects, but the terahertz wave itself was not visualized. In contrast, our purpose is to directly observe the electric field profile of the wave. The first experiments of this type were done in $1999 .{ }^{10}$ Since then numerous experiments have employed imaging to study terahertz waves. ${ }^{11}$ These techniques have been used to study terahertz generation, ${ }^{12-14}$ and the interaction of terahertz waves with various structures. ${ }^{15-19}$ Recent advancements in imaging ${ }^{20-22}$ have made it possible to collect high resolution, in-focus videos of terahertz waves interacting with a variety of fabricated structures. These recent developments were incorporated in this study to make the images and videos as clear and understandable as possible.

Section II explains how terahertz waves are generated and detected and how videos of propagating waves are captured. It also explains how we produce samples with structures such as slits and waveguides. This background will help readers to better understand the experiments, but is not necessary for understanding the principles demonstrated by the videos which are presented in Sec. III. The first experiment highlights interference by crossing two beams, and the second emphasizes diffraction resulting from a wave passing through a single slit. The next three experiments (Young's double-slit experiment, a transmission grating, and a reflection grating) display the combined effects of diffraction and interference. The sixth experiment shows focusing of a curved phase front. The final two experiments display the capabilities and effects of waveguiding. The variety of geometries in these experiments demonstrates several important optical phenomena and indicates how further instructive examples could be compiled.

\section{EXPERIMENTAL}

\section{A. Generation of terahertz electromagnetic waves}

Terahertz waves are generated by focusing short, intense laser pulses into a nonlinear crystal. The laser pulses are produced by an "ultrafast" titanium:sapphire laser and amplifier, ${ }^{23}$ with a center wavelength of $800 \mathrm{~nm}$, a duration of 100 femtoseconds $\left(10^{-13} \mathrm{~s}\right)$, and about $1 \mathrm{~mJ}$ of energy. Such high-energy, short duration pulses have very large peak intensities of around $3 \times 10^{11} \mathrm{~W} / \mathrm{cm}^{2}$, which allow them to exert significant forces on the ions in a crystal lattice, displacing them from their equilibrium positions. This class of phenomena falls under the umbrella of nonlinear optics, for which there are many textbooks. ${ }^{24,25}$ The key result here is that a small part of the energy from the short, high-frequency ( $\sim 375 \mathrm{THz}$ ) optical pump pulse is converted into a short, low-frequency $(\sim 0.5 \mathrm{THz})$ pulse which is typically several picoseconds in duration.

In the present experiments we used the nonlinear crystal lithium niobate, $\mathrm{LiNbO}_{3}$. The nonlinear physics of terahertz generation in $\mathrm{LiNbO}_{3}$ has been well studied. ${ }^{6,26}$ In this crystal, impulsive stimulated Raman scattering is responsible for converting some of the optical frequency light to terahertz frequencies. A terahertz wave generated in $\mathrm{LiNbO}_{3}$ propagates at a large angle relative to the optical pump pulse which produced it, ${ }^{6}$ because the speed of the optical pulse is much higher than the speed of the terahertz pulse. The effect, known as the Cherenkov effect, is similar to that of a boat rushing through water faster than the speed of water waves, where the wake left by the boat propagates mainly sideways relative to the boat speed in a typical inverted "V" pattern. In the experiments described below, we restrict the propagation angle to be strictly perpendicular to the direction of the optical pulse by guiding the terahertz wave with a very thin slab of $\mathrm{LiNbO}_{3}$. The $\mathrm{LiNbO}_{3}$ slab, which has a high index of refraction and is only $50 \mu \mathrm{m}$ thick, acts as a waveguide that traps the wave within it through total internal reflection. (Optical fibers are also examples of waveguides; even when they are bent, light propagates through them without escaping into the air.) The optical pump propagates perpendicularly to the slab surface and the generated terahertz wave travels in the plane of the slab [see Fig. 1(a)]. Many characteristics of the wave can be controlled by changing the pump pulse characteristics. ${ }^{13,14}$ We chose the center frequency and duration of the terahertz pulse that most clearly demonstrate the optical principles involved.

\section{B. Imaging of terahertz electromagnetic waves}

A terahertz wave propagates at the speed of light in the sample, and must be detected using an extremely fast method. We used a second ultrafast optical pulse called the probe pulse. We converted $800 \mathrm{~nm}$ light to $532 \mathrm{~nm}$ light for
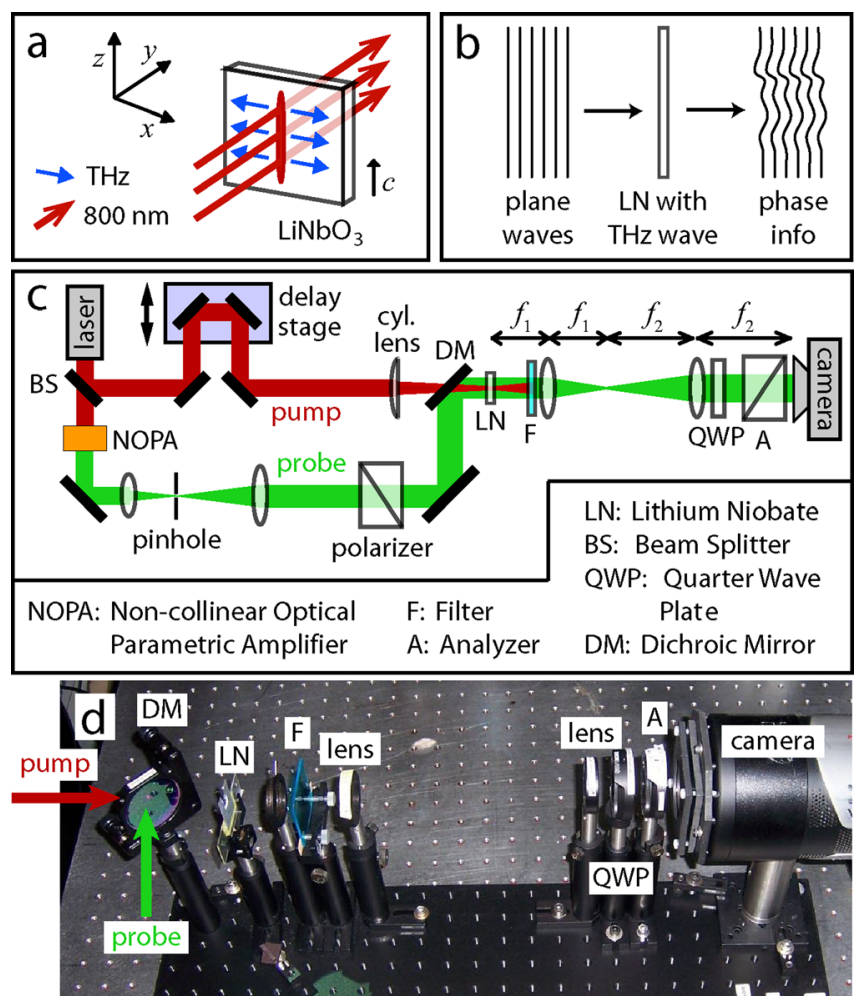

Fig. 1. (a) The generation geometry: the pump laser pulse propagates orthogonal to the slab surface, and the generated terahertz wave propagates perpendicularly to the pump pulse in the plane of the slab. (b) The vertical lines to the left of the sample represent the optical plane wave phase fronts of the incoming probe pulse. After the sample, the phase fronts are distorted due to the presence of the terahertz wave. (c) A simplified layout for the entire experiment. The short pulses from the laser are split by the $90 \%$ reflecting beam splitter. The pump beam is routed through a delay stage so it can be delayed relative to the probe. The sample is placed between crossed polarizers so that any terahertz-induced birefringence in the sample is recorded on the camera, providing an image of the terahertz field profile. (d) A photograph of the detection part of the experimental setup corresponding the diagram in (c). The drilled holes on the breadboard and optical table are spaced 1 in apart. 
the probe, so that it could be separated from the pump after the sample with a single spectral filter. The probe light was spatially filtered to improve the mode quality and expanded [see Fig. 1(c)] so that it would illuminate the entire sample. The probe is sensitive to a terahertz wave-induced change in the sample. Because $\mathrm{LiNbO}_{3}$ is an electro-optic crystal, an applied electric field changes its index of refraction such that $n=n_{0}+a E$, where $n_{0}$ is the index of refraction in the absence of an electric field, $E$ is the applied field, and $a$ is a crystal-dependent constant. In reality, the index and field are related by a tensor which depends on the angle of the field, crystal, and probe light, ${ }^{11}$ but these details are not essential for understanding this work. The terahertz wave can be thought of as a spatially dependent electric field, $E_{\mathrm{THz}}(x, z)$, which can be approximated as constant through the depth of the crystal due to the waveguiding geometry. ${ }^{22}$ Thus, the terahertz field induces a spatially dependent index of refraction in the sample given by

$$
n(x, z)=n_{0}+a E_{\mathrm{THz}}(x, z) .
$$

The probe pulse is an expanded laser beam that can be approximated as a plane wave. As the plane waves propagate through the sample, they develop a phase shift proportional to the index of refraction [see Fig. 1(b)]. Where the refractive index has been increased, the probe light speed is reduced, and the probe phase lags behind the phase of the unperturbed plane wave. Where the refractive index has been decreased, the probe light speed is increased and the probe phase runs ahead. Thus, immediately after the sample, a phase pattern is imposed on the beam, and the induced phase shift at any point in the $x-z$ plane is directly proportional to the terahertz electric field at that point.

The sample is imaged onto the camera using a 2-lens, 4-focal length imaging system. If nothing more were done, the $\mathrm{LiNbO}_{3}$ crystal would be imaged perfectly onto the camera but no terahertz wave would be detected. The wave would not be visible because it modulates the phase of the probe light, and the camera is sensitive only to the intensity. Thus, an optical technique must be used to perform phase-toamplitude conversion. Several methods can do this conversion, ${ }^{22}$ and here we use the polarization gating method.

A simplified geometry containing the key conceptual features of the polarization gating setup is shown in Fig. 1(c). Polarization gating detects a change in the birefringence of the $\mathrm{LiNbO}_{3}$ sample induced by the terahertz field, which changes the index of refraction of different crystal axes by different amounts. To perform polarization gating, the sample must be placed between two crossed polarizers. The first polarizer sets the probe polarization to $45^{\circ}$, and the terahertzinduced change in the polarization state of the probe changes the transmission through the second analyzing polarizer. The quarter-wave plate sets the unperturbed transmission to $50 \%$, and ensures that a positive phase shift will be detected as a positive amplitude change and vice versa. The detected intensity for the diagram in Fig. 1(c) is given by ${ }^{22}$

$$
I(x, z)=I_{0}(x, z)[1+\sin (\Delta \phi(x, z))] .
$$

$I_{0}$ is the unperturbed intensity measured when there is no terahertz wave present (for example, when the pump pulse is blocked), and $\Delta \phi$ is the difference in the induced phase shift experienced by vertically and horizontally polarized light in the presence of the terahertz wave. This phase difference is given by

$$
\begin{aligned}
\Delta \phi(x, z) & =-2 \pi \frac{d}{\lambda_{\mathrm{pr}}}\left[\Delta n_{v}(x, z)-\Delta n_{h}(x, z)\right] \\
& =-2 \pi \frac{d}{\lambda_{\mathrm{pr}}} a^{\prime} E_{\mathrm{THz}}(x, z)
\end{aligned}
$$

where $d$ is the slab thickness, $\lambda_{\mathrm{pr}}$ is the probe wavelength, $\Delta n_{v}$ and $\Delta n_{h}$ are the terahertz-induced change in index for vertically and horizontally polarized light, and $a^{\prime}$ is a material dependent constant. By measuring both $I(x, z)$ and $I_{0}(x, z)$, the spatially dependent terahertz field can be determined.

\section{Capturing videos of terahertz waves: the pump-probe method}

The imaging method in Sec. II B captures the spatial profile of a terahertz wave at one moment in time. To collect a video of the wave as it propagates and evolves, the pumpprobe method is required. The short laser pulse coming out of the laser is split by a beamsplitter [see Fig. 1(c)]. Most of the energy goes into the pump, which is used to launch the terahertz wave, and some goes into the probe, which is used to image the terahertz wave that the pump launched. Both pulses propagate in air at the speed of light, and the time it takes for each pulse to reach the $\mathrm{LiNbO}_{3}$ sample is given by the path length divided by $c$. Thus, the amount of time the probe arrives after the pump is

$$
\tau=\left(d_{\mathrm{pr}}-d_{\mathrm{pu}}\right) / c
$$

where $d_{\mathrm{pr}}$ and $d_{\mathrm{pu}}$ are the respective optical path lengths of the probe and pump pulses. When the path lengths of both the pump and probe arms are exactly the same, as shown in the first frame of Fig. 2, the probe measures the moment of terahertz wave generation. The image in the lower right shows the signal that would be collected on the camera for point source generation (pump focused into the crystal). As the pump path length is shortened (by moving the motorized delay stage in Fig. 2 toward the probe beam), $\tau$ is increased and the terahertz wave has longer to evolve before its spatial profile is captured by the probe. To make a video, an image is captured as we have described for a series of time delays. Each delay corresponds to one frame in the video.

The general technique of increasing the delay between two pulses is called the pump-probe method. It is widely used to study phenomena that happen on time scales faster than $\sim 1$ nanosecond, that is, the attosecond, femtosecond, and picosecond regimes. For this method to work, the same experiment must be performed hundreds of times (at least once for each time delay), and the results must be reproducible to build a consistent picture. We rely on the fact that nearly identical laser pulses launch the terahertz waves the same way every time without damaging or otherwise modifying the sample. Because we have to make the measurement at many time delays, and the results of many pulses at each delay are averaged to reduce noise, acquiring these videos often takes many hours.

\section{Structuring the sample using laser machining}

To recreate classic experiments in the thin $\mathrm{LiNbO}_{3}$ slab, it is necessary to introduce structures such as mirrors, slits, and 


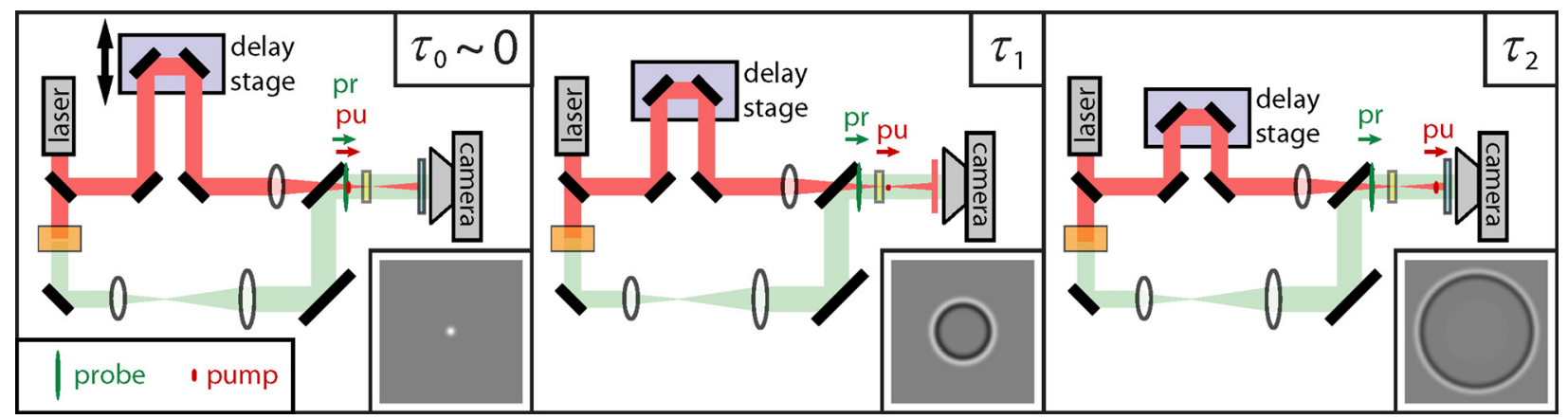

Fig. 2. Simplified pictures of the experimental setup showing that as the delay stage moves, the time delay between pump and probe pulses, shown as small ellipses near the sample, is changed. The two pulses arrive at about the same time in the first frame, and as the stage shortens the pump path length, the wave launched by the pump pulse has longer to evolve before the probe pulse arrives. The images inset in the lower right of each frame show the terahertz wave that would be captured on the camera at each time delay if the wave were launched by a point source pump, expanding outward much like the water wave that is created when a stone is thrown into a pond.

waveguides directly into the slab. These structures are introduced by cutting air gaps into the crystal slab. Because $\mathrm{LiNbO}_{3}$ has a very high index of refraction $(n \sim 5.1)$ for terahertz-frequency light, reflection off air interfaces is very efficient. The total internal reflection angle is only $11^{\circ}$ in bulk $\mathrm{LiNbO}_{3}$, so terahertz waves are reflected off even gently tilted surfaces with $100 \%$ efficiency. We used femtosecond laser machining to cut air gaps in the crystal slab. ${ }^{27}$ In this method, a short-pulsed laser is tightly focused into the sample using a microscope objective. The sample is moved along a specified trajectory using computer controlled motors, and the laser cuts through the crystal slab as it moves. If the sample is moved in a circle, a circular hole is cut out of the slab. Any pattern can be programmed into the computer and cut into the sample, making it easy to generate interesting structures.

\section{RESULTS: CLASSIC EXPERIMENTS VISUALIZED}

Each of Sec. III A-H describes a different experimental geometry. Each geometry has one or two video clips in the supporting online material, and select frames are shown in the associated figure. In all the videos and figures, the darker-gray region is $\mathrm{LiNbO}_{3}$ crystal, and the uniform, lighter-gray region is a machined air gap cut as described in Sec. II D. In Figs. 3-6, there is a theoretical prediction for the field pattern in addition to the experimental data. The theoretical prediction assumes a continuous wave input with a well defined frequency. The theory shows the root mean square electric field: $E_{\mathrm{rms}}(x, z)=\left[\nu \int_{\nu t=0}^{1} E^{2}(x, z, t) d t\right]^{1 / 2}$, where $\nu$ is the frequency. $E_{\mathrm{rms}}$ is the square root of the time averaged intensity, which is what would typically be observed visually in a simple experiment with a $\mathrm{HeNe}$ laser and diffractive optic in an introductory physics lab.

\section{A. Crossing two beams}

In this simple experiment, two beams, which are well approximated by plane waves, are crossed to make an interference pattern [see Fig. 3(a)]. The many applications of this geometry include crossing beams to generate a periodic pattern in optical lithograph ${ }^{28}$ or a transient grating for a wide array of experiments. ${ }^{29}$ The spatial period of the intensity pattern, $\Lambda$, depends only on the wavelength in the material, $\lambda$, and the crossing angle, $\theta$ :

$$
\Lambda=\frac{\lambda}{2 \sin (\theta / 2)}
$$

Figure 3(a) shows the amplitude pattern predicted for crossing two beams, one propagating from upper left to lower right in the figure, and the other propagating from lower left to upper right. Figures 3(b)-3(e) contain frames from Video 1 showing two beams crossing. Initially, a terahertz wave with several optical cycles reflects off two tilted surfaces. The reflected waves cross and form an interference pattern with horizontal nodes as predicted. As the waves propagate, the phase within the intensity maxima evolves but the horizontal nodes in the interference pattern remain unchanged. By the end of the video, the two beams have separated and the interference pattern has mostly disappeared. The angle between the beams is $60^{\circ}$. Equation (5) predicts that the

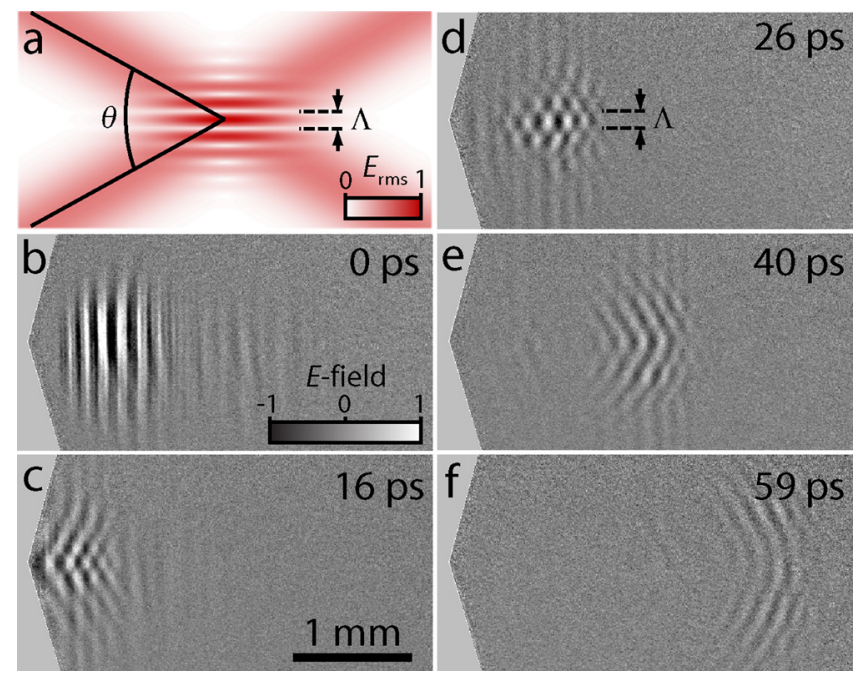

Fig. 3. Beams crossing at an angle of $60^{\circ}$. (a) The theoretical time-averaged $E$-field amplitude, $|E|=\sqrt{I}$ which results from crossing two continuouswave beams. (b)-(f) Frames from Video 1 at a sequence of time delays. The uniform, light gray region on the left of each frame is an air gap that has been cut into the thin $\mathrm{LiNbO}_{3}$ slab. The terahertz wave is clearly visible in each frame of the video, with white corresponding to a positive electric field and black corresponding to a negative electric field. The wave is propagating to the left in (b). In (c) the wave has just reflected off the air interface and the upper half and lower halves of the initial pulse have formed two beams that are crossing at an angle of $60^{\circ}$. In (d) the two beams are fully overlapped, and by (f) they have almost completely separated (video online) [URL: http://dx.doi.org/10.1119/1.3652698.1]. 


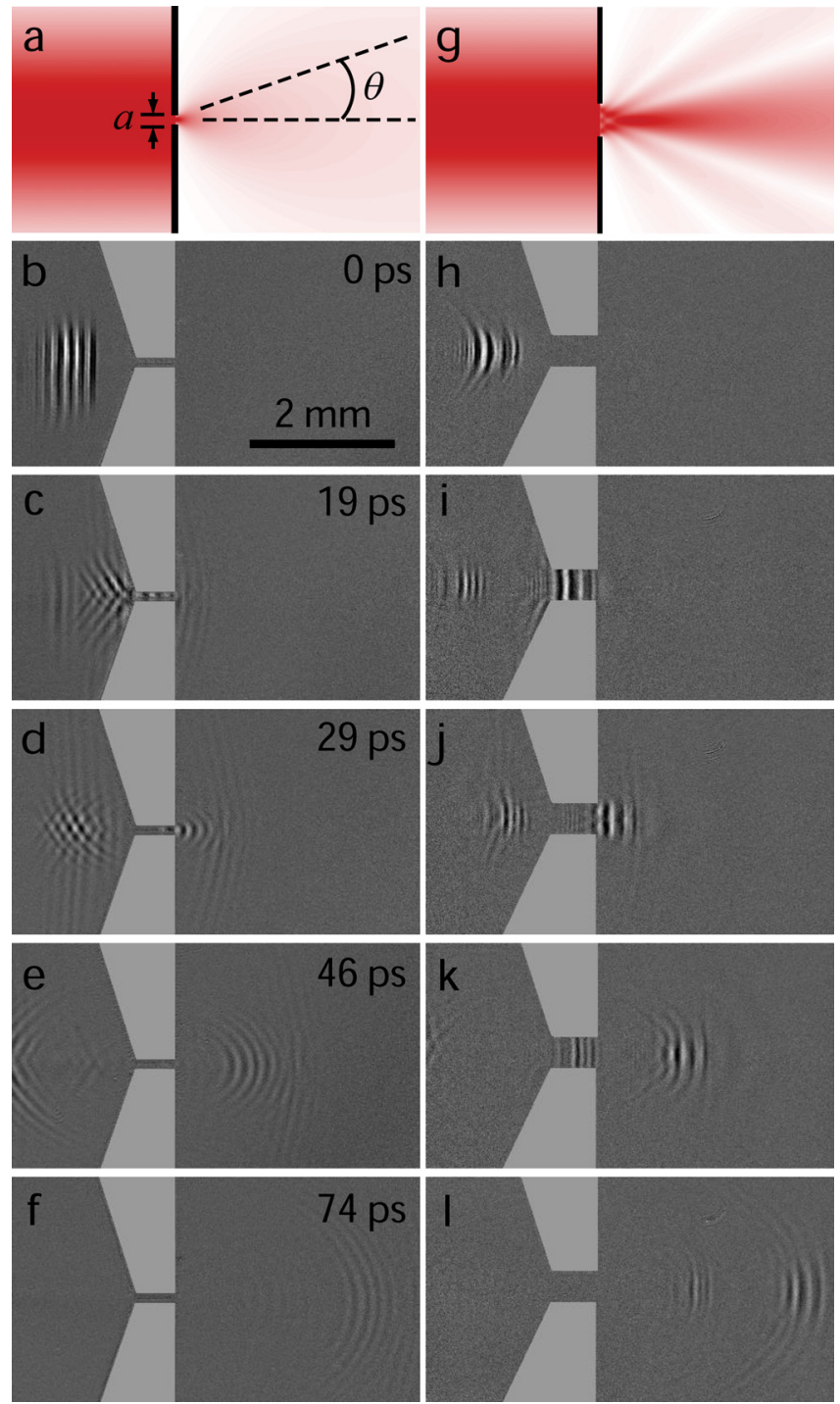

Fig. 4. Diffraction through a single slit. (a)-(f) are for a wave propagating through a slit whose width is $4 \lambda / 5$ (video online) [URL: http://dx.doi.org/ $10.1119 / 1.3652698 .2]$. (g)-(l) are for a slit whose width is $3 \lambda$ (video online) [URL: http://dx.doi.org/10.1119/1.3652698.3]. (a) and (g) Predictions of the time-averaged electric field amplitudes for a continuous wave beam entering from the left. (b)-(f) and (h)-(1) Frames from Videos 2 and 3, respectively, showing experimentally measured electric field profiles for a short terahertz pulse entering from the left. Adjacent images have the same time delays. The uniform light gray regions are air gaps that have been cut into the crystal.

period of the intensity pattern is equal to the terahertz wavelength $(\sim 160 \mu \mathrm{m})$, which agrees with the experiment. Note that Fig. 3(a) shows the time-averaged rms value of the field, and hence the time-dependent field oscillations between positive and negative values which are apparent in Figs. 3(b)-3(e) do not appear in Fig. 3(a).

\section{B. Diffraction through one slit}

Diffraction through a single slit is a classic wave mechanics phenomenon. In this experiment, a plane wave is incident on a small slit, and the diffraction pattern of the transmitted light is observed. Although it is possible to determine the behavior near the slit (a complicated interference pattern) using the Fresnel Kirchhoff formula, ${ }^{30}$ the treatment is mathematically too complex for an introductory course. It is possible to determine the behavior far from the slit, the

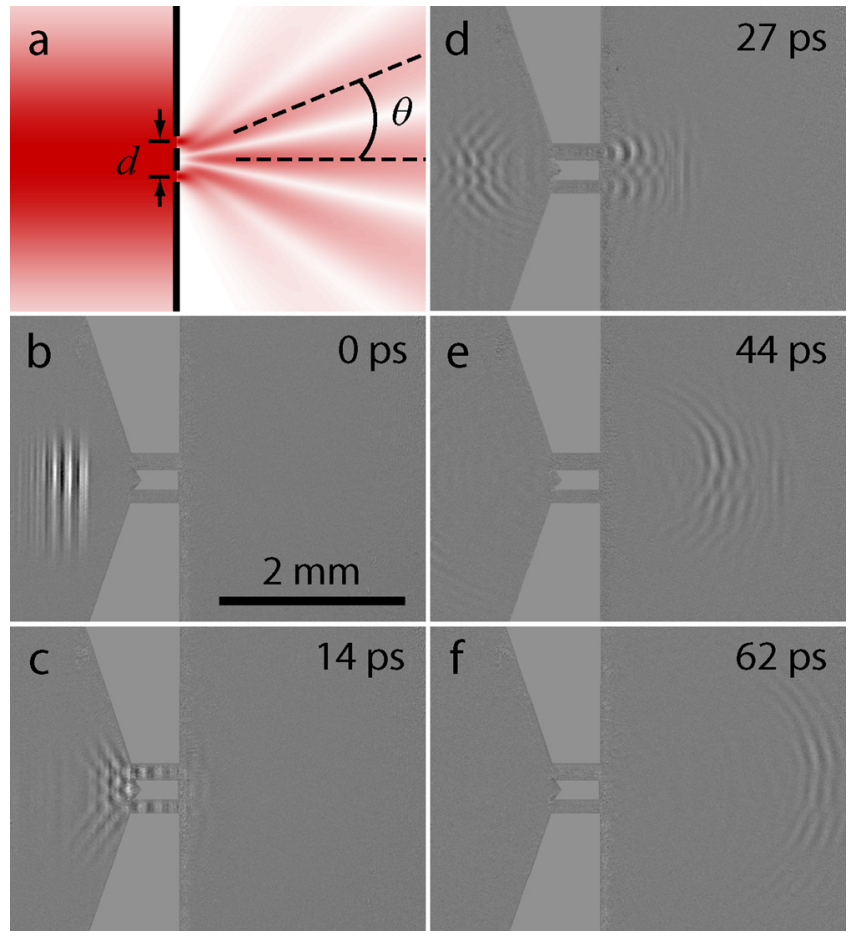

Fig. 5. Two-slit diffraction. (a) The theoretical, time-averaged electric field amplitude. (b)-(f) Frames from Video 4. The uniform, slightly lighter gray region is an air gap. The wave initially propagates to the right, and a significant fraction is reflected off the air gap while part propagates through the slits. The waves reflected or transmitted from the two slits interfere with one another as the waves propagate away from the slits. The slit width is $\lambda$ and the slit center to center spacing is $5 \lambda / 2$ (video online) [URL: http:// dx.doi.org/10.1119/1.3652698.4]

Fraunhoffer diffraction limit, using simple methods. ${ }^{31}$ The far-field intensity pattern as a function of angle is

$$
I=I_{0}\left[\frac{\sin (\gamma)}{\gamma}\right]^{2},
$$

with $\gamma=\pi a \sin (\theta) / \lambda$. The slit width $a$ and the emission angle $\theta$ are defined in Fig. 4(a). If the slit width is less than the wavelength $\lambda$, then the slit acts like a point source, which yields the dipole emission pattern, and only the main central lobe is observed. This case is shown in Figs. 4(a)-4(f) and Video 2, where the slit width is $a=4 \lambda / 5$. Figures 4(a) and $4(\mathrm{~g})$ show the root-mean square $E$-field patterns calculated exactly using the Fresnel Kirchhoff formula, ${ }^{32}$ a method which is also used in Figs. 5(a) and 6(a). In Figs. 4(g) $-4(\mathrm{l})$ and Video 3, the slit width is $a=3 \lambda$. As predicted by Eq. (6), there are nodes in the emission pattern for this larger gap spacing. The central lobe contains most of the energy and diverges more slowly than in the smaller gap spacing.

\section{Diffraction through two slits}

Double-slit diffraction is another classic experiment. A plane wave is incident on a pair of slits of width $a$ separated by a distance $d$ [see Fig. 5(a)]. Light diffracts from each slit as described in Sec. III B, and the light from the two sources interfere with each other. It is complicated to analytically describe the behavior near the slits, but the far-field behavior can be calculated with relative ease, ${ }^{31}$ and is given by 


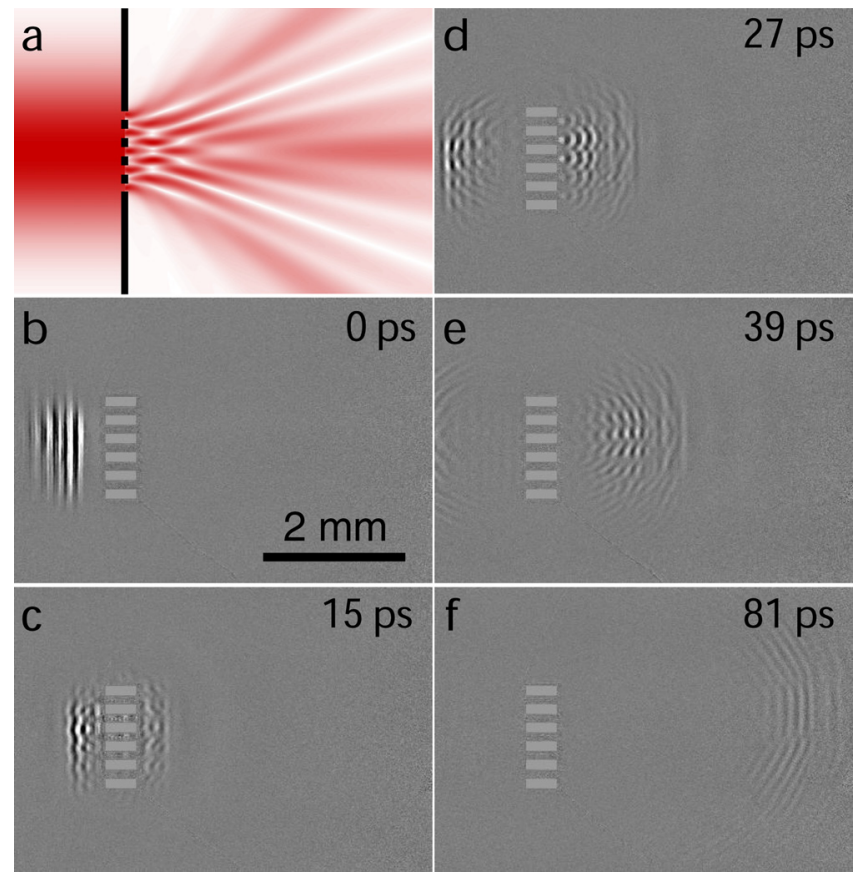

Fig. 6. Five-slit diffraction. (a) The root-mean square $E$-field for a continuous wave laser beam. (b)-(f) Frames from Video 5. The uniform light gray regions are air gaps. Part of the initially rightward propagating wave reflects off the air gaps and part is transmitted through the slits. Wave propagation reveals the mechanism through which interference close to the slits evolves into the farfield emission pattern. The slit period is $d=5 \lambda / 3$ and the slit width is $a=5 \lambda / 6$ (video online) [URL: http://dx.doi.org/10.1119/1.3652698.5].

$$
I=I_{0}\left[\frac{\sin (\gamma)}{\gamma}\right]^{2} \cos ^{2}(\delta),
$$

where $\delta=\pi d \sin (\theta) / \lambda$ has a definition to similar $\gamma$ in Sec. III B. Equation (7) gives a periodic interference pattern multiplied by the single slit diffraction pattern given in Sec. III B. In Fig. 5 and Video 4 , the slit spacing is $d=2.5 \lambda$ and the slit width is $a=\lambda$. Because $a$ is close to $\lambda$, the diffraction part of the equation is smooth and slowly decaying as one moves away from the center. The modulation results from interference between the outputs from the two slits. The video shows the interference evolving close to the slits and eventually developing into the far-field pattern described by Eq. (7).

\section{Diffraction through $\mathbf{N}$ slits}

As more slits are added, the intensity maxima become narrower and brighter. Between the bright peaks are a set of nulls and significantly weaker maxima [see Fig. 6(a)]. The exact expression in the far-field is given by ${ }^{30}$

$$
I=I_{0}\left[\frac{\sin (\gamma)}{\gamma}\right]^{2}\left[\frac{\sin (N \delta)}{N \sin (\delta)}\right]^{2} .
$$

As in Sec. III C, the single-slit envelope function is multiplied by a factor describing the interference. The limit of an infinite number of slits is a transmission grating, in which the weak peaks disappear completely and there are a set of narrow, well defined diffraction directions. Our experiment, where roughly five slits are illuminated, shows an intermediate case between the double slit and a transmission grating.
Although it is difficult to see all the minor maxima in the data [see Figs. 6(b)-6(f) and Video 5], it is interesting to see how a complicated interference pattern close to the slit evolves into a simpler diffraction pattern in the far-field.

\section{E. Reflection grating}

Of the geometries we have described, the reflection grating is the most used as a component in typical optical experiments. Because the points of diffraction are so close together, a monochromatic source generates very welldefined diffraction orders. The well-known grating equation relating the diffraction directions and groove spacing is ${ }^{33}$

$$
m \lambda=d\left[\sin (\alpha)+\sin \left(\beta_{m}\right)\right]
$$

where $m$ is an integer, $d$ is the groove period on the grating, $\alpha$ is the angle of incidence, and $\beta_{m}$ is the angle of diffraction as defined in Fig. 7(a). The convention used to determine the signs of the angles is indicated by + and - in Fig. 7(a). Any periodic surface with period $d$ will follow Eq. (9). Propagating diffraction orders will exist if $|m \lambda / d|<2$, when the sum of the sines does not exceed 2. Although the existence of the diffraction orders is determined by Eq. (9), the distribution of intensity into the different orders is determined by the detailed profile of the periodic structure.

Many gratings are created with a saw-tooth surface pattern. This pattern selectively sends most of the energy into a specific diffraction order, and efficiencies in excess of $90 \%$ are routinely achieved. ${ }^{33}$ In a grating with such a saw-tooth pattern, the blaze angle, or the angle of the tooth surface, is set so that if the surface of each tooth were extended, the incident and diffracted beam would obey the law of
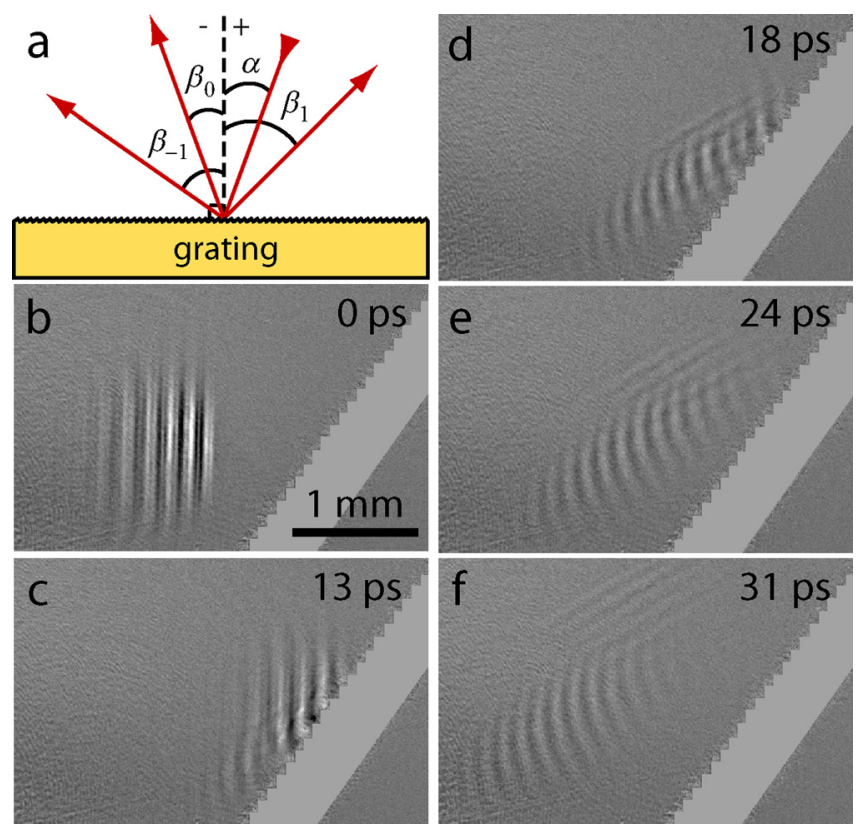

Fig. 7. A reflection grating. (a) The angles for the grating geometry. (b)-(f) Frames from Video 6 . The uniform light gray region is an air gap that has been carved into the crystal. The grating period, angle, and blaze have been optimized for the Littrow configuration, shown here. The initially rightward propagating wave is split into the 0 th order beam (the reflection that would be produced by a smooth surface) which propagates up to the left and the 1st order beam which is diffracted directly back along the input path (video online) [URL: http://dx.doi.org/10.1119/1.3652698.6]. 

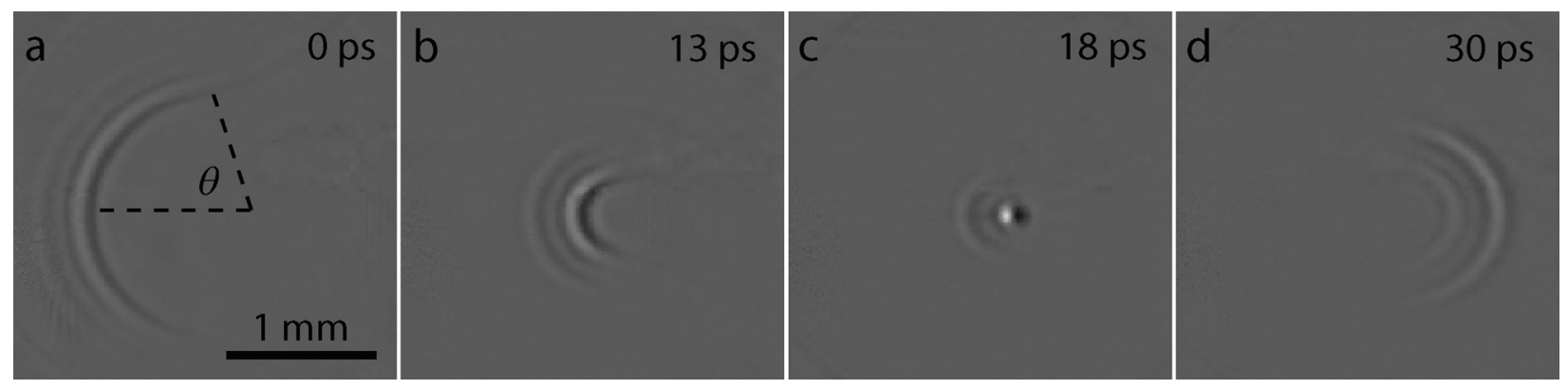

Fig. 8. A focusing wave. (a)-(d) Frames from Video 7. An axicon focuses the pump pulse into a 5 mm thick slab of LiTaO 3 to generate a curved phase front. The rightward propagating wave is focused to a diffraction-limited spot in (c), before it starts diverging (video online) [URL: http://dx.doi.org/10.1119/1.3652698.7].

reflection. In effect, each facet or tooth behaves like a subwavelength mirror. For an overview of different types of gratings and grating designs, see Ref. 33. The Littrow configuration, where the angle of incidence is equal to the angle of reflection $\left(\beta_{1}=\alpha\right)$, is very common due to its high efficiency, and is shown in Fig. 7.

To prepare a grating in the Littrow configuration, the period of the steps are designed and the grating is angled such that the $m=1$ diffraction order propagates directly back along the incoming direction. The grating is blazed such that the surface of each step is vertical and the sub-wavelength mirrors reflect directly backward, improving the efficiency. Figures 7(b)-7(f) show frames from Video 6 . The wave initially propagates to the right and diffracts off the grating. The zero order reflection, visible in Figs. 7(d)-7(f), propagates up and to the left. The first-order diffracted beam propagates directly back along the incoming direction. The intensity front of the diffracted wave is tilted, a feature that would not be visible if the light source were continuous instead of a short pulse. In fact, a grating was used to tilt the visible pump pulse which generated the terahertz wave in Video $6 .^{14}$

\section{F. Wave focusing}

The simple geometry here follows a focusing electromagnetic wave. A curved terahertz wave is generated using an axicon, a glass cone, in combination with a lens to focus the pump beam to a ring on the sample. ${ }^{16}$ The curvature of the generated phase front is similar to the phase front resulting from transmission through a lens or reflection off a curved mirror. When collecting this video, the right side of the ring of light was blocked to form a semicircular wave pattern. Figure 8 and Video 7 show the focusing terahertz wave. The wave collapses to a tight spot, enhancing and localizing the electric field, and then quickly diverges. The spot size at the focus is given by

$$
d \approx \frac{\lambda_{0}}{2 \mathrm{NA}}=\frac{\lambda_{0}}{2 n \sin (\theta)},
$$

where $\lambda_{0}$ is the wavelength in free space, NA is the numerical aperture, $n$ is the index of refraction, and $\theta$ is the angle defined in Fig. 8. Lenses and mirrors with large acceptance angles and thus large numerical apertures, such as microscope objectives, can focus to (or resolve) very small spots. In the experiment shown in Fig. $8, n=6.15$ and $\theta$ is almost $90^{\circ}$, yielding an NA of about 6 , much larger than that of common optical lenses and mirrors. The wavelength observed in the experiment is $\lambda_{0} / n$, and thus the diameter of the focused spot is about half the observed wavelength. One other interesting phenomenon noticeable in Video 7 is the Gouy phase shift - the change in wave polarity as it passes through the focus. ${ }^{12,34}$ Direct visualization of this effect is important for understanding how fields change in the focus of an electromagnetic wave.

\section{G. Y-coupler}

A Y-coupler is a simple structure that demonstrates waveguiding and interference. Air gaps (light gray in Fig. 9 and Video 8) were cut into the slab leaving $\mathrm{LiNbO}_{3}$ in the shape of a Y. A tall, uniform terahertz wave is launched initially and a small section of it is incident on the top and bottom arms of the Y. These sections of the wave are waveguided, trapped inside the high index of refraction material due to total internal reflection. (The terahertz waves inside a lithium niobate slab are already in a planar waveguide, but because the confinement is along the same direction as the optical pump and probe beam propagation, preventing the terahertz waves from escaping the plane of the lithium niobate, the images do not directly illustrate waveguiding. In the present case, waveguiding is apparent in the images because confinement prevents the waves from propagating along some directions within the image plane.) The terahertz waves travel down each arm of the $\mathrm{Y}$ until they meet in the center and interfere. The wave resulting from their superposition is further guided down the stem of the $\mathrm{Y}$ until it is emitted. The

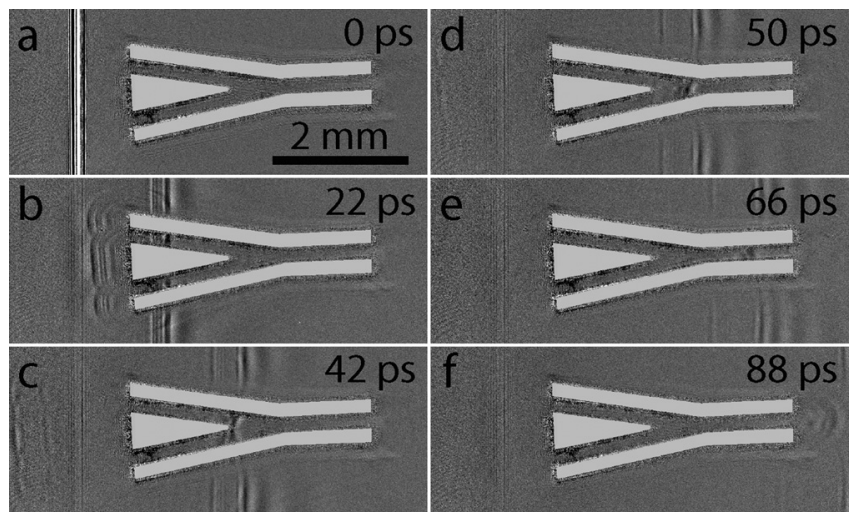

Fig. 9. A Y-coupler. (a)-(f) Frames from Video 8. The uniform light gray region is an air-gap carved into a $50 \mu \mathrm{m}$ slab of $\mathrm{LiNbO}_{3}$. The rightwardpropagating terahertz wave is initially guided down each arm of a Y. When the waves meet in the middle they interfere, and are further guided down the stem of the Y. When the wave is emitted at the end it starts to diverge due to diffraction (video online) [URL: http://dx.doi.org/10.1119/1.3652698.8]. 
idea of using a high-index material to trap and guide light is important because it is responsible for the functioning of optical fibers and other photonic components.

\section{H. Dielectric slab waveguide propagation}

As mentioned briefly in Sec. II, terahertz waves were guided in a slab of $\mathrm{LiNbO}_{3}$ for most of our measurements. The waveguiding was useful because it confined the terahertz wave to a thin region that could be imaged. The final experiment demonstrates how waveguiding behavior can be visualized and studied in the imaging setup. Before showing the experimental results, a quick review of dielectric slab waveguides, also called planar waveguides, will be presented. Unlike the material from Sec. III A-G which would usually be covered in an introductory physics class, the material discussed in this section is more advanced and is usually discussed in an advanced undergraduate or graduate optics class. In spite of the disparity in complexity, we discuss waveguiding here because the experiment provides a clear qualitative and quantitative visualization of waveguiding behavior, which could be very helpful in a more advanced class. We will discuss the results and solutions to the dielectric waveguide but not their derivation, which can be found in many textbooks such as Ref. 35 .

A dielectric slab waveguide consists of a high-index core, such as $\mathrm{LiNbO}_{3}$, surrounded by a low-index material, such as air. In a waveguide, the profile of the electric and magnetic field down the depth of the crystal [along $y$ in Fig. 1(a)] does not change as the wave propagates. This behavior is similar to an eigenmode in quantum mechanics, with time $t$ from quantum mechanics replaced by the distance along the propagation direction $x$; the solutions for the transverse electric (TE) mode of the dielectric slab waveguide are exactly the same as those for a particle in a box with finite potential walls. Our discussion will be limited to the TE modes, which have the electric field parallel to the slab surface and perpendicular to the propagation direction, because they are the only modes present in this experiment, although others can be generated. ${ }^{36}$ For a specific propagation constant, $\beta=2 \pi / \lambda$, with $\lambda$ the wavelength along the direction of propagation, there are multiple modes with different numbers of nodes. The 0th mode has no nodes, the 1 st mode has one node, etc. Profiles of the first three modes in a $50 \mu \mathrm{m}$ thick $\mathrm{LiNbO}_{3}$ slab in air with $\beta=100 \mathrm{rad} / \mathrm{mm}(\lambda=63 \mu \mathrm{m})$ are shown in Fig. 10(a). The solutions are harmonic inside the high-index material (cosine for the even, symmetric modes and sine for the odd, antisymmetric modes). In contrast, the solutions are evanescent and exponentially decaying in the low-index material outside.

The wavelength inside the high-index material and the decay length in the low-index material are frequency dependent. Figure 10(b) shows how the profile of the lowest mode changes with frequency. At low frequencies, that is, long wavelengths, the evanescent decay length is very long and most of the wave's energy is in the air, while at shorter wavelengths, the evanescent field decays very quickly and most of the energy is localized in the $\mathrm{LiNbO}_{3}$.

One common way to plot this frequency- and modedependent behavior is with a dispersion curve [see Fig. 10(c)]. A dispersion curve shows the frequency (or energy) as a function of wave vector. The two thin dashed lines show the dispersion curve for air, $\omega=c \beta$, and for bulk $\mathrm{LiNbO}_{3}, \omega=c \beta / n$. The dispersion curve for the waveguide shows that at low frequencies, the wave behaves more like a wave in air because so much of the energy is in the evanescent field. At high frequencies, the wave behaves more like a wave in bulk $\mathrm{LiNbO}_{3}$ because most of the energy is in the $\mathrm{LiNbO}_{3}$ slab. The lowest mode extends
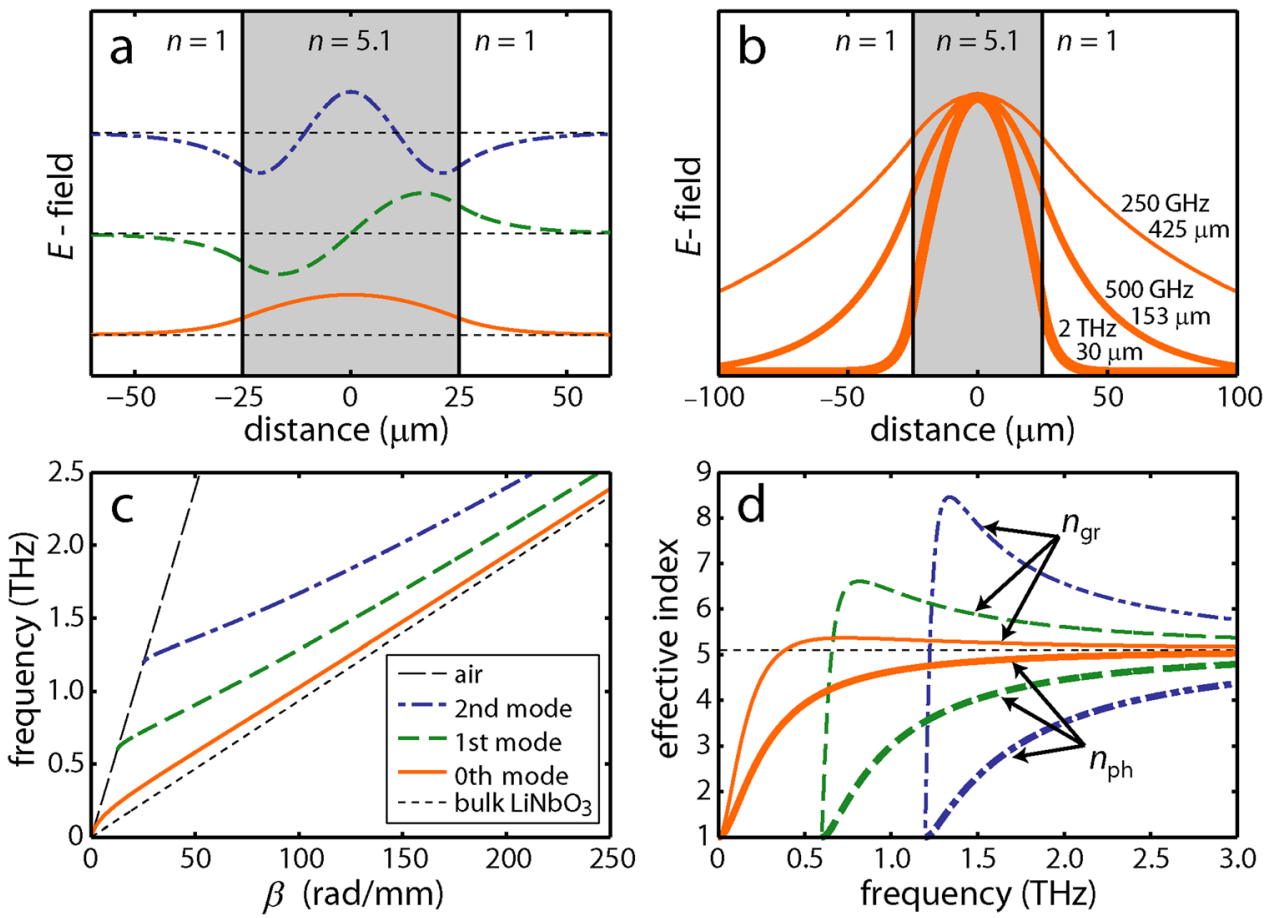

Fig. 10. Analytical waveguide solutions for TE modes in a dielectric slab waveguide. (a) The $E$-field profile of the first three modes with a propagation constant $\beta=100 \mathrm{rad} / \mathrm{mm}$. (b) The $E$-field profiles for the lowest mode at three frequencies. The wavelength along the propagation direction is indicated below the frequency. (c) The dispersion curves for the first three modes. (d) The effective group index (upper three, thinner curves) and effective phase index (lower three, thicker curves) for the waveguide. 


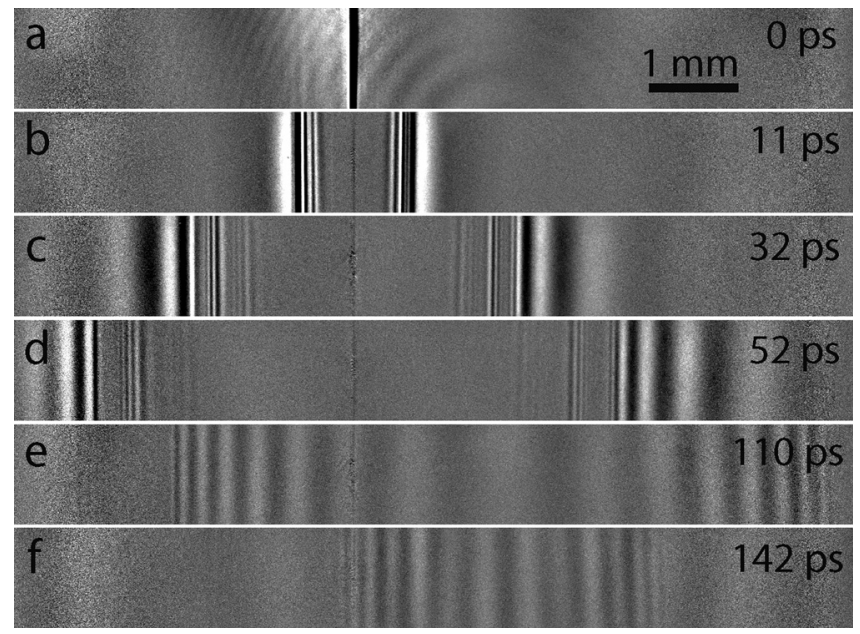

Fig. 11. Images of a broadband, guided terahertz wave. (a)-(f) Frames from Video 9 depicting broadband terahertz waves propagating in an unstructured $\mathrm{LiNbO}_{3}$ crystal. Counterpropagating waves are launched by a cylindrically focused "line" of pump pulse light in (a), and they have just separated in (b). The first three waveguide modes are clearly visible in (c) and (d). After frame (d) the wave reflects off of the crystal edges so the waves are propagating toward one another in (e); they have begun to overlap and interfere in (f) (video online) [URL: http://dx.doi.org/10.1119/1.3652698.9].

all the way to zero frequency and wave vector. The higher modes have cutoff frequencies below which there are no waves with the appropriate number of nodes bound in the slab.

Another way to display the same information is with the effective indices of refraction, both the phase index, $n_{\mathrm{ph}}$ and the group index, $n_{\mathrm{gr}}$. The phase index is defined as $n_{\mathrm{ph}}(\omega)=c \beta(\omega) / \omega$. The velocity at which a single phase front moves, the phase velocity, is given by $v_{\mathrm{ph}}=c / n_{\mathrm{ph}}$, and the wavelength along the direction of propagation is given by $\lambda=2 \pi c /\left(\omega n_{\mathrm{ph}}\right)$. The lower three, thicker curves in Fig. 10(d) show the phase index as a function of frequency for the lowest three modes. $n_{\mathrm{ph}}$ transitions smoothly from the index of air to the index of bulk $\mathrm{LiNbO}_{3}$. The velocity at which a pulse or wave-packet travels, the group velocity, is given by $v_{\mathrm{gr}}=c / n_{\mathrm{gr}}$ $=d \omega / d \beta$. Although the group index starts close to one for each mode, it quickly increases until it is larger than the group index for the bulk material [see the upper three curves in Fig. 10(d)]. The large group index means that wave packets of the appropriate frequency travel more slowly than in the bulk material. As the frequency increases and the slab becomes thick compared with the wavelength, both $n_{\mathrm{gr}}$ and $n_{\mathrm{ph}}$ approach the value of the bulk index of the dielectric - the group index from above and the phase index from below.

In the experiment described here, terahertz waves are excited in a plain, unstructured waveguide by focusing the pump beam to a line in the $\mathrm{LiNbO}_{3}$ slab. This geometry excites a singlecycle terahertz wavepacket which contains many frequencies and the first three waveguide modes. Figure 11 shows frames from Video 9. Initially two counter-propagating single-cycle waves are launched. As the waves propagate, the first three waveguide modes separate due to their differences in group velocity. The three modes are clearly visible as distinct wavepackets in Figs. 11(c) and 11(d). By looking just at the 0th waveguide mode, it is easy to see that the lower frequency, longer wavelength components travel faster than the highfrequency components, a consequence of the fact that the lowfrequency, long-wavelength components are propagating mostly in air. The different velocities for different frequencies leads to the pulse broadening and becoming "chirped," that is,
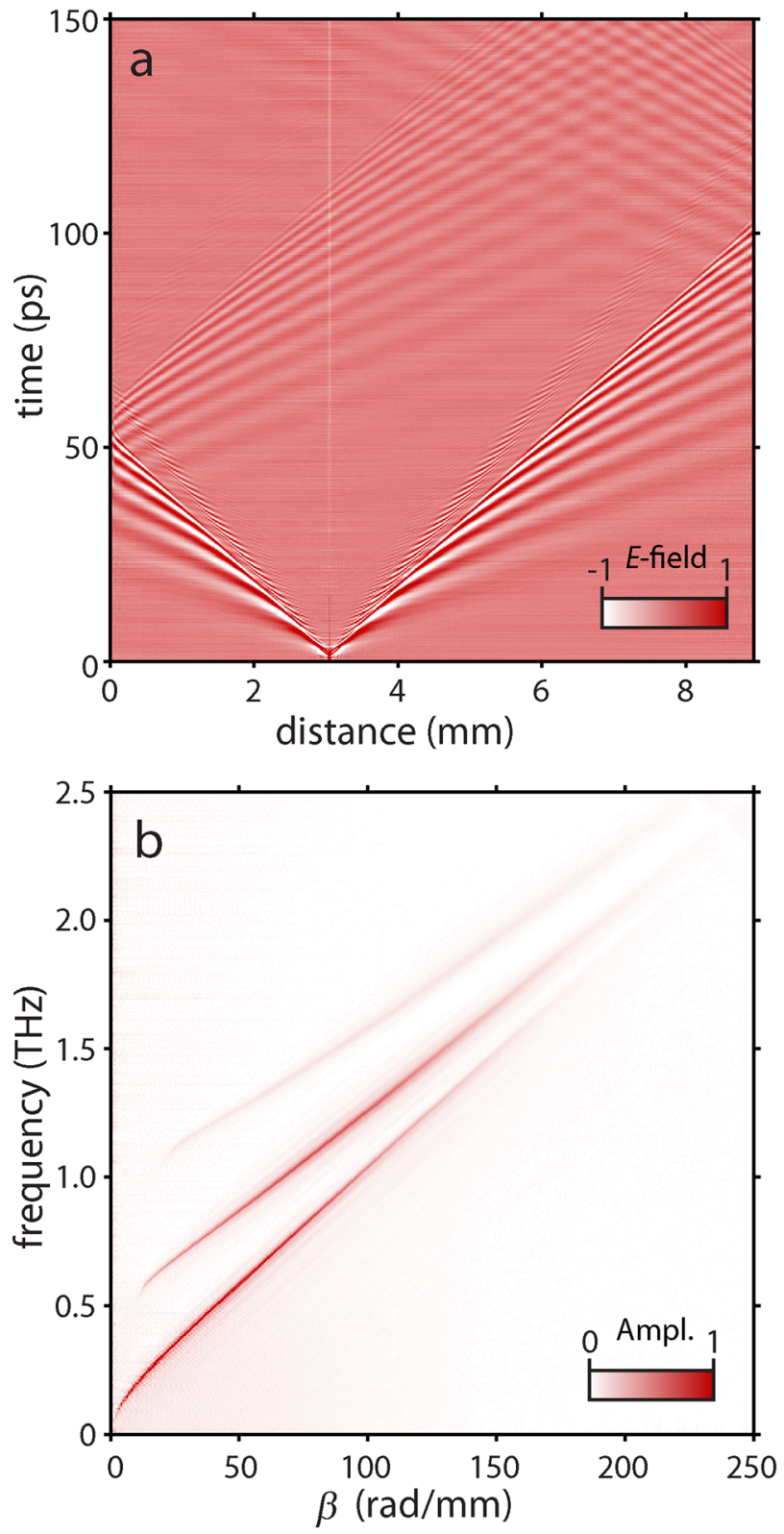

Fig. 12. Two-dimensional plots of a waveguided terahertz wave. (a) A space-time plot showing the evolution of a broadband terahertz pulse in an unstructured slab waveguide. The two-dimensional Fourier transform of (a) yields the experimentally determined dispersion curves shown in (b).

having a time-dependent frequency. The waves reflect off the crystal edge after frame (d) in Fig. 11 and eventually cross, at which point the two counter-propagating waves form a standing wave [see Fig. 11(f)]. Counter-propagation is a limiting case of Eq. (5) with $\theta=\pi$. The interference pattern that is formed by the intersecting beams is striking because the fringe spacing starts out relatively large and gradually becomes smaller as the shorter-wavelength components arrive at the region of intersection between the two beams.

In every frame in Video 9, the $E$-field is uniform along the vertical $(z)$ direction. As a result, it is possible to compress each $m \times n$ picture into a $1 \times n$ array by averaging over the vertical dimension with no information loss. The $1 \times n$ array is the electric field profile as a function of horizontal position at one time delay. The compression is performed for each frame in the video, and each $1 \times n$ array is placed into one row of a matrix, one above the other, in time order so that from bottom to top the 
field profiles at successively later times are stored. The resulting space-time plot shows the full evolution of the wave as it propagates [see Fig. 12(a)]. In this single figure, it is possible to see the modes separate, the pulses begin to become chirped, and the reflection and interference of the waves. In this plot, the slope is directly proportional to the index: $m=\Delta t / \Delta x=1 / v=n / c$. The slope of the wavepacket is given by the group index, and the slope of the phase front is given by the phase index. It is clear from Fig. 12(a) that the group index of the second mode is higher than that of the first mode, but the phase index is lower. The two-dimensional Fourier transform of the matrix in Fig. 12(a) enables a quantitative comparison with analytical theory. The time dimension transforms to frequency and the spatial dimension transforms to the propagation constant, so the result of the Fourier transform is a direct experimental determination of the dispersion curve. Figure 12(b) shows this matrix, which is in good agreement with the analytical theory shown in Fig. 10(c)..$^{21}$ The direct observation of guided electromagnetic waves helps develop intuition for the formation of discrete modes and waveguide dispersion, effects common to optical fibers and many other waveguide geometries.

\section{CONCLUSIONS}

The experimental technique we have described enables visualization of electromagnetic waves propagating at the speed of light. We hope that this paper and its accompanying videos can help bring contemporary optical research into the classroom and provide a valuable tool in an introductory and intermediate physics curriculum. We also hope that other researchers in terahertz optics and spectroscopy will contribute additional figures and videos to a growing library of illustrations of waves in various contexts. To facilitate the growth of such an online library, we would gladly add videos from other researchers (with appropriate attribution) to the collection already on our website. ${ }^{37}$

\section{ACKNOWLEDGMENTS}

This work was supported in part by National Science Foundation Grant Nos. ECCS-0824185 and CHE-0616939, and a NSF Graduate Research Fellowship to C.A. Werley.

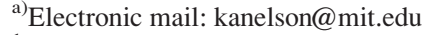

${ }^{1}$ Y. J. Dori and J. Belcher, "How does technology-enabled active learning affect undergraduate students' understanding of electromagnetism concepts?,” J. Learn. Sci. 14, 243-279 (2005).

${ }^{2}$ Computational Electrodynamics: The Finite-Difference Time-Domain Method, 3rd ed., edited by A. Taflove and S. C. Hagness (Artech House, Boston, MA, 2005)

${ }^{3}$ M. Sipos and B. G. Thompson, "Electrodynamics on a grid: The finitedifference time-domain method applied to optics and cloaking," Am. J. Phys. 76, 464-469 (2008).

${ }^{4}$ D. W. Ward, E. R. Statz, N. S. Stoyanov, and K. A. Nelson, "Simulation of Phonon-Polariton Propagation in Ferroelectric $\mathrm{LiNbO}_{3}$ Crystals," in Engineered Porosity for Microphotonics and Plasmonics, MRS Symposium Proceedings, 2003, Vol. 762, pp. C11.60.1-6.

${ }^{5}$ J. D. Joannopoulos, S. G. Johnson, J. N. Winn, and R. D. Meade, Photonic Crystals, Molding the Flow of Light, 2nd ed. (Princeton U. P., Princeton, NJ, 2008), Chap. 2.

${ }^{6} \mathrm{D}$. Auston and M. Nuss, "Electrooptic generation and detection of femtosecond electrical transients," IEEE J. Quantum Electron. 24, 184-197 (1988).

${ }^{7}$ D. Grischkowsky, S. Keiding, M. van Exter, and Ch. Fattinger, "Far-infrared time-domain spectroscopy with terahertz beams of dielectrics and semiconductors," J. Opt. Soc. Am. B 7, 2006-2015 (1990).

${ }^{8} \mathrm{Q}$. Wu, T. D. Hewitt, and X.-C. Zhang, "Two-dimensional electro-optic
} imaging of THz beams," Appl. Phys. Lett. 69, 1026-1028 (1996).
${ }^{9}$ Z. Jiang and X.-C. Zhang, "Terahertz imaging via electrooptic effect," IEEE Trans. Microwave Theory Tech. 47, 2644-2650 (1999).

${ }^{10}$ R. M. Koehl, S. Adachi, and K. A. Nelson, "Direct visualization of collective wavepacket dynamics," J. Phys. Chem. A 103, 10260-10267 (1999).

${ }^{11}$ T. Feurer, N. S. Stoyanov, D. W. Ward, J. C. Vaughan, E. R. Statz, and K. A. Nelson, “Terahertz polaritonics," Annu. Rev. Mater. Res. 37, 317-350 (2007).

${ }^{12}$ T. Feurer, N. S. Stoyanov, D. W. Ward, and K. A. Nelson, "Direct visualization of the Gouy phase by focusing phonon polaritons," Phys. Rev. Lett. 88, 257402-1-4 (2002).

${ }^{13}$ T. Feurer, J. C. Vaughan, and K. A. Nelson, "Spatiotemporal coherent control of lattice vibrational waves," Science 299, 374-377 (2003).

${ }^{14}$ K.-H. Lin, C. A. Werley, and K. A. Nelson, "Generation of multicycle $\mathrm{THz}$ phonon-polariton waves in a planar waveguide by tilted optical pulse fronts," Appl. Phys. Lett. 95, 103304 (2009).

${ }^{15}$ N. S. Stoyanov, T. Feurer, D. W. Ward, and K. A. Nelson, "Integrated diffractive THz elements," Appl. Phys. Lett. 82, 674-676 (2002).

${ }^{16}$ N. S. Stoyanov, D. W. Ward, T. Feurer, and K. A. Nelson, "Terahertz polariton propagation in patterned materials," Nature Mater. 1, 95-98 (2002).

${ }^{17}$ N. S. Stoyanov, T. Feurer, D. W. Ward, E. R. Statz, and K. A. Nelson, "Direct visualization of a polariton resonator in the THz regime," Opt. Express 12, 2387-2396 (2004).

${ }^{18} \mathrm{P}$. Peier, S. Pilz, and T. Feurer, "Time-resolved coherent imaging of a THz multilayer response,” J. Opt. Soc. Am. B 26, 1649-1655 (2009).

${ }^{19}$ P. Peier, H. Merbold, V. Pahinin, K. A. Nelson, and T. Feurer, "Imaging of $\mathrm{THz}$ waves in $2 \mathrm{D}$ photonic crystal structures embedded in a slab waveguide," New J. Phys. 12, 013014 (2010).

${ }^{20}$ P. Peier, S. Pilz, F. Müller, K. A. Nelson, and T. Feurer, "Analysis of phase contrast imaging of terahertz phonon-polaritons," J. Opt. Soc. Am. B 25, B70-B75 (2008).

${ }^{21}$ Q. Wu, C. A. Werley, K.-H. Lin, A. Dorn, M. G. Bawendi, and K. A. Nelson, "Quantitative phase contrast imaging of $\mathrm{THz}$ electric fields in a dielectric waveguide," Opt. Express 17, 9219-9225 (2009).

${ }^{22}$ C. A. Werley, Q. Wu, K.-H. Lin, C. R. Tait, A. Dorn, and K. A. Nelson, "Comparison of phase-sensitive imaging techniques for studying terahertz waves in structured $\mathrm{LiNbO}_{3}$," J. Opt. Soc. Am. B 27, 2350-2359 (2010).

${ }^{23} \mathrm{D}$. Strickland and G. Mourou, "Compression of amplified chirped optical pulses," Opt. Commun. 56, 219-221 (1985).

${ }^{24}$ R. W. Boyd, Nonlinear Optics, 3rd ed. (Elsevier, Burlington, MA, 2008).

${ }^{25}$ Y. R. Shen, The Principles of Nonlinear Optics (John Wiley \& Sons, Hoboken, NJ, 2003).

${ }^{26}$ T. P. Dougherty, G. P. Wiederrecht, and K. A. Nelson, "Impulsive stimulated Raman scattering experiments in the polariton regime," J. Opt. Soc. Am. 9, 2179-2189 (1992).

${ }^{27}$ D. W. Ward, E. R. Statz, and K. A. Nelson, "Fabrication of polaritonic structures in $\mathrm{LiNbO}_{3}$ and $\mathrm{LiTaO}_{3}$ using femtosecond laser machining," Appl. Phys. A 86, 49-54 (2007).

${ }^{28} \mathrm{C}$. Mack, Fundamental Principles of Optical Lithography: The Science of Microfabrication (John Wiley \& Sons, Hoboken, NJ, 2007).

${ }^{29}$ H. J. Eichler, P. Günter, and D. W. Pohl, Laser-Induced Dynamic Gratings (Springer-Verlag, Berlin, 1986).

${ }^{30} \mathrm{G}$. R. Fowles, Introduction to Modern Optics, 2nd ed. (Dover Publications, Mineola, NY, 1989), Chap. 5.

${ }^{31}$ R. Resnik, D. Halliday, and K. S. Krane, Phyiscs, 5th ed. (John Wiley \& Sons, New York, 2002).

${ }^{32}$ We rederived the Fresnel Kirchhoff formula following the derivation in Ref. 30 , but in two dimensions instead of three because wave behavior in the waveguide follows two-dimensional behavior. Making the additional assumption that plane waves are incident on the aperture, the Green's function is $G(x, z)=-Y_{0}(2 \pi \rho / \lambda)[x / \rho+1]$ with $Y_{0}$ a Bessel function of the second kind and $\rho^{2}=x^{2}+z^{2}$ the radius in two dimensions. The $E$-field can then be calculated by performing a numerical convolution of the aperture function with the Green's function $E(x, z, t)=[A(z) \otimes G(x, z)] \exp (-i \omega t)$.

${ }^{33}$ C. Palmer, Diffraction Grating Handbook, 5th ed. (Newport, Rochester, NY, 2005).

${ }^{34}$ L. G. Gouy, "Sur une propriété nouvelle des ondes lumineuses," C. R. Acad. Sci. Paris 110, 1251-1253 (1890).

${ }^{35}$ N. J. Cronin, Microwave and Optical Waveguides (Institute of Physics, Philadelphia, PA, 1995).

${ }^{36}$ C. Yang, Q. Wu, J. Xu, K. A. Nelson, and C. A. Werley, "Experimental and theoretical analysis of THz-frequency, direction-dependent, phonon polariton modes in a subwavelength, anisotropic slab waveguide," Opt. Express 18, 26351-26364 (2010).

${ }^{37}$ nelson.mit.edu/ under "movietheater." 